\title{
Ribotype differences between clinical and environmental isolates of Burkholderia pseudomallei
}

\author{
S. TRAKULSOMBOON*†, D. A. B. DANCE $\ddagger$, M. D. SMITH $\S^{* *} \|$, N. J. WHITE\&\| and T. L. PITT* \\ *Laboratory of Hospital Infection, Central Public Health Laboratory, 61 Colindale Avenue, London NW9 5HT, \\ $\dagger$ Division of Infectious Disease, Department of Medicine, Faculty of Medicine, Siriraj Hospital, Mahidol \\ University, Bangkok 10700, Thailand, $\ddagger$ Public Health Laboratory, Derriford Hospital, Plymouth PL6 8DH, \\ $\S$ Faculty of Tropical Medicine, Mahidol University, Bangkok 10400, Thailand and \|Centre for Tropical Medicine, \\ Nuffield Department of Clinical Medicine, John Radcliffe Hospital, Headington, Oxford OX3 9DU
}

\begin{abstract}
Burkholderia pseudomallei is isolated frequently from the soil in regions where the disease melioidosis occurs. However, recent surveys in Thailand have shown that the frequency of isolation of the organism from soil samples is not directly related to the incidence of melioidosis in an area. To determine whether strain populations of $B$. pseudomallei prevalent in soil are gentypically related to strains causing clinical disease, rRNA Bam HI restriction fragment length polymorphisms (RFLP) of 139 soil environmental isolates and 228 human isolates were compared. Two groups of ribotype patterns were found. Group I comprised 37 different ribotype patterns which were characterised by five to eight hybridisation bands of $2.8->23 \mathrm{~kb}$. All of these ribotypes were identified among the clinical isolates, and 18 of them were also found in 59 environmental isolates. Group II was represented by 12 ribotypes found only in environmental strains. These ribotype patterns comprised one to five bands in the size range 9- $>23 \mathrm{~kb}$. All but one of the 73 isolates in this group grew on a minimal medium supplemented with L-arabinose. In contrast, only $3 \%$ of the 66 isolates from the environment with group I ribotype patterns could utilise this sugar as their sole energy source. These findings suggest that $B$. pseudomallei strains that utilise arabinose constitute a population that is genetically distinct from other environmental and clinical strains.
\end{abstract}

\section{Introduction}

Melioidosis is an infection caused by Burkholderia pseudomallei. Despite the apparent ubiquity of $B$. pseudomallei in the soils of endemic areas of Southeast Asia, there is great variability in the incidence of melioidosis in different regions [1]. This may be due to host susceptibility related to conditions such as diabetes mellitus, a well recognised risk factor [2], and differences in occupational exposure to the organism. Differences in bacterial virulence might also contribute to the geographic distribution of melioidosis.

A recent study by Wuthiekanun et al. [3] suggests that

Received 15 Aug. 1996; accepted 14 Nov. 1996.

Corresponding author: Dr T. L. Pitt.

** Present address: Public Health Laboratory, Musgrove Park Hospital, Taunton TA1 5DB. although clinical and environmental isolates of $B$. pseudomallei from Thailand are morphologically similar and antigenically indistinguishable, two populations of the species are revealed by extended biochemical testing. They identified two different biotypes of $B$. pseudomallei that were characterised by two phenotypes based on their ability to utilise the sugar L-arabinose as a sole energy source for growth. This study suggested that the biotype differences are associated with the disease-producing potential of the bacteria for man as arabinose positive $\left(\mathrm{ara}^{+}\right)$isolates were found exclusively in the environment and not from patients with melioidosis, while $\mathrm{ara}^{-}$isolates were isolated from both man and the environment. The question arises as to whether these varieties of $B$. pseudomallei are genomically related or show sufficient differences to warrant further investigation of species identity. Therefore, the variation in rRNA gene loci of isolates of both phenotypes was examined to determine their degree of genetic relatedness. 


\section{Materials and methods}

\section{Bacterial isolates}

A total of 367 isolates of $B$. pseudomallei was examined; 139 isolates were from the environment (133 soil, five water, one manure) and their details including sites and geographical origin are given in Table 1. All but 20 of the environmental isolates were from Thailand; the non-Thai isolates originated from nine different countries. The remaining 228 isolates were from a collection of strains from cases of human melioidosis worldwide, the great majority of which were from Southeast Asia.

All isolates were presumptively identified as $B$. pseudomallei by their reaction with Gram's stain and colony morphology on Ashdown's selective medium, production of oxidase and resistance to gentamicin (10 $\mu \mathrm{g}$ disk) and colistin $(10 \mu \mathrm{g})$ [4]. Species identity was confirmed by API 20NE profiles (bioMérieux, Marcy, France). Arabinose utilisation was determined by growth on minimal salts agar [5] containing Larabinose $0.2 \%$. Ethanol assimilation was tested by the growth of isolates and production of acid on ethanol $1 \%$ in an ammonium salts based medium [6].

\section{Ribotyping}

Ribotyping of isolates was performed as described by Kaufmann et al. [7]. Chromosomal DNA was extracted with guanidium thiocyanate from the 48 -h growth on nutrient agar and digested with Bam $\mathrm{HI}$ restriction endonuclease. The DNA fragments were separated by electrophoresis in agarose gel and transferred to a nylon membrane. The membrane was treated with a biotin-labelled cDNA of rRNA from Escherichia coli (Boehringer Mannheim, Germany) and DNA hybridisation bands were visualised colorimetrically with the BlueGene kit (Gibco-BRL, Uxbridge). The migration distance of each DNA band on the membrane was measured manually and molecular size was calculated with reference to bacteriophage lambda DNA digested with Hind III and labelled with biotin (Gibco-BRL). Each profile was differentiated according to the number and position of DNA bands and was assigned an arbitrary number. Pair-wise comparisons of similarity were made by the Dice coefficient [8] and cluster analysis was performed by the unweighted pair group matching band average (UPGMA) and displayed as a dendrogram with the aid of a computer program (MVSP; Kovach Computing Services, Pentraeth, Anglesey).

\section{Statistical test}

Associations between the source of isolates, their ribotype group and utilisation of substrates for growth were tested for statistical significance by the $\chi^{2}$ test.

\section{Results}

The migration distance of each rDNA hybridisation band was measured for all isolates of $B$. pseudomallei from clinical melioidosis and the environment, and their molecular size was calculated with reference to lambda markers. Among the 228 human isolates, 15 distinct band positions were identified, ranging in size from 2.8 to $>23 \mathrm{~kb}$; most isolates were characterised by three to eight bands, and a total of 37 different patterns were distinguishable by visual comparison (Fig. 1). These patterns were termed group I, and 18 of them were also found among 59 of the 139 environmental isolates examined. Seven isolates from the environment had unique ribotypes not found among human isolates and correspondingly 18 human isolates had unique ribotypes that were not found amongst the environmental isolates. Two individual ribotype patterns (types 1 and 3) predominated in both clinical and environmental isolates in group I (Table 2).

A second group of ribotype patterns was recognised exclusively in environmental isolates. This group was characterised by one to five bands in the size range $9->23 \mathrm{~kb}$ (Fig. 1) and only one band position coincided with the 15 bands on which the 37 patterns in group I were based. At least 12 different patterns formed this group II and were distinguished from group I ribotypes by the prefix A (Table 2). Three patterns predominated among the 73 isolates represented by 8,27 and 10 isolates, respectively, and the DNA of 11 isolates was not digested by Bam HI.

Fig. 1 shows that cluster analysis of pair-wise comparisons of ribotypes within group I revealed an overall similarity of $62 \%$ and most of these patterns differed by two or more band positions. However, groups I and II ribotype clusters were only $25 \%$ related to each other. On the basis of simple matching for the presence or absence of a particular band in the gel track, group II isolates were markedly unrelated to each other and a percentage similarity as low as $30 \%$ was required to group the five isolates together. This was due in the main to the fact that only eight band positions were identified within group II ribotypes and despite their limited size distribution, many isolates differed from each other by three to four bands.

Table 3 shows the percentage of environmental and clinical isolates that utilised arabinose or ethanol, or both, and their distribution in groups I and II ribotype patterns. Overall, $74(53.2 \%)$ of 139 environmental isolates utilised arabinose as a sole energy source, but only two of 66 isolates of group I ribotype patterns were ara $^{+}$compared with 72 of 73 groups II isolates $\left(\chi^{2}=126.33, \quad p<0.0001\right)$. Similarly, significantly more isolates in group II (63 of 73) assimilated ethanol compared with group I $\left(4 / 26 ; \chi^{2}=42.63\right.$, $\mathrm{p}<0.0001)$. Thus, the great majority of ribotype group I isolates from the environment were similar to 
Table 1. Sites, geographical origin and dates of isolation of environmental isolates of $B$. pseudomallei

\begin{tabular}{|c|c|c|c|c|c|c|c|c|c|}
\hline No. & Ref. no & Site & Origin & Date & No. & Ref. no. & Site & Origin & Date \\
\hline 1 & 978-C & Soil & Australia & $\ldots$ & 70 & $\mathrm{D} 260 \mathrm{~S} 3 / 30$ & Soil & Australia & $\ldots$ \\
\hline 2 & Pingy & Water & Thailand S & & 71 & D30 S3/40 & Soil & Australia & $\ldots$ \\
\hline 3 & EE091 & Soil & Thailand NE & 1990 & 72 & D34 S3/50 & Soil & Australia & $\ldots$ \\
\hline 4 & E250 & Soil & Thailand NE & 1992 & 73 & D75 S3/50 & Soil & Australia & $\ldots$ \\
\hline 5 & E183 & Soil & Thailand NE & 1992 & 74 & D85 S2/30 & Soil & Australia & $\ldots$ \\
\hline 6 & E198 & Soil & Thailand NE & 1992 & 75 & D109 S3/40 & Soil & Australia & $\ldots$ \\
\hline 7 & E212 & Soil & Thailand NE & 1992 & 76 & D112 S3/30 & Soil & Australia & $\ldots$ \\
\hline 8 & E010 & Soil & Thailand NE & 1990 & 77 & E13 & Soil & Thailand NE & 1991 \\
\hline 9 & E086 & Soil & Thailand NE & 1990 & 78 & E06 & Soil & Thailand NE & 1990 \\
\hline 10 & E090 & Soil & Thailand NE & 1990 & 79 & E08 & Soil & Thailand NE & 1990 \\
\hline 11 & Somredee & Water & Thailand S & & 80 & E13 & Soil & Thailand NE & 1991 \\
\hline 12 & E021 & Soil & Thailand NE & 1990 & 81 & E15 & Soil & Thailand NE & 1991 \\
\hline 13 & E043 & Soil & Thailand NE & 1990 & 82 & E16 & Soil & Thailand NE & 1991 \\
\hline 14 & E044 & Soil & Thailand NE & 1990 & 83 & E20 & Soil & Thailand NE & 1992 \\
\hline 15 & E195 & soil & Thailand NE & 1992 & 84 & E255 & Soil & Thailand NE & $\ldots$ \\
\hline 16 & E208 & Soil & Thailand NE & 1992 & 85 & E257 & Soil & Thailand NE & \\
\hline 17 & E213 & Soil & Thailand NE & 1992 & 86 & E053 & Soil & Thailand NE & 1990 \\
\hline 18 & E218 & Soil & Thailand NE & 1992 & 87 & E064 & Soil & Thailand NE & 1990 \\
\hline 19 & E223 & Soil & Thailand NE & 1992 & 88 & E072 & Soil & Thailand NE & 1990 \\
\hline 20 & E225 & Soil & Thailand NE & 1992 & 89 & E074 & Soil & Thailand NE & 1990 \\
\hline 21 & E231 & Soil & Thailand NE & 1992 & 90 & E094 & Soil & Thailand NE & 1991 \\
\hline 22 & E235 & Soil & Thailand NE & 1992 & 91 & E099 & Soil & Thailand NE & 1991 \\
\hline 23 & E243 & Soil & Thailand NE & 1992 & 92 & E100 & Soil & Thailand NE & 1991 \\
\hline 24 & E249 & Soil & Thailand NE & 1992 & 93 & E102 & Soil & Thailand NE & 1991 \\
\hline 25 & E023 & Soil & Thailand NE & 1990 & 94 & E107 & Soil & Thailand NE & 1991 \\
\hline 26 & E190 & Soil & Thailand NE & 1992 & 95 & E127 & Soil & Thailand NE & 1991 \\
\hline 27 & E215 & Soil & Thailand NE & 1992 & 96 & E149 & Soil & Thailand NE & 1991 \\
\hline 28 & E039 & Soil & Thailand NE & 1990 & 97 & E178 & Soil & Thailand NE & 1991 \\
\hline 29 & Taib & Water & Thailand S & & 98 & E260 & Soil & Thailand C & 1993 \\
\hline 30 & E182 & Soil & Thailand NE & 1992 & 99 & E261 & Soil & Thailand $\mathrm{C}$ & 1993 \\
\hline 31 & E242 & Soil & Thailand NE & 1992 & 100 & E263 & Soil & Thailand $\mathrm{C}$ & 1993 \\
\hline 32 & E245 & Soil & Thailand NE & 1992 & 101 & E267 & Soil & Thailand C & 1993 \\
\hline 33 & E237 & Soil & Thailand NE & 1992 & 102 & E229 & Soil & Thailand NE & 1992 \\
\hline 34 & E220 & Soil & Thailand NE & 1992 & 103 & E201 & Soil & Thailand NE & 1992 \\
\hline 35 & E222 & Soil & Thailand NE & 1992 & 104 & E216 & Soil & Thailand NE & 1992 \\
\hline 36 & 93-D2 & Soil & Australia & $\ldots$ & 105 & R179 & Soil & Thailand NE & 1991 \\
\hline 37 & 149 & Soil & Australia & & 106 & E251 & Soil & Thailand NE & \\
\hline 38 & E181 & Soil & Thailand NE & 1992 & 107 & E048 & Soil & Thailand NE & 1990 \\
\hline 39 & E187 & Soil & Thailand NE & 1992 & 108 & E075 & Soil & Thailand NE & 1990 \\
\hline 40 & E015 & Soil & Thailand NE & 1990 & 109 & E078 & Soil & Thailand NE & 1990 \\
\hline 41 & E244 & Soil & Thailand NE & 1992 & 110 & E082 & Soil & Thailand NE & 1990 \\
\hline 42 & 74101 & Water & Iran & 1974 & 111 & E116 & Soil & Thailand NE & 1991 \\
\hline 43 & E087 & Soil & Thailand NE & 1990 & 112 & E171 & Soil & Thailand NE & 1991 \\
\hline 44 & E240 & Soil & Thailand NE & 1992 & 113 & E175 & Soil & Thailand NE & 1991 \\
\hline 45 & E038 & Soil & Thailand NE & 1990 & 114 & E125 & Soil & Thailand NE & 1991 \\
\hline 46 & E041 & Soil & Thailand NE & 1990 & 115 & E049 & Soil & Thailand NE & 1990 \\
\hline 47 & E139 & Soil & Thailand NE & 1991 & 116 & E143 & Soil & Thailand NE & 1991 \\
\hline 48 & E227 & Soil & Thailand NE & 1992 & 117 & E184 & Soil & Thailand NE & 1992 \\
\hline 49 & E238 & Soil & Thailand NE & 1992 & 118 & E262 & Soil & Thailand C & 1993 \\
\hline 50 & E224 & Soil & Thailand NE & 1992 & 119 & E266 & Soil & Thailand C & 1993 \\
\hline 51 & E037 & Soil & Thailand NE & 1990 & 120 & E180 & Soil & Thailand NE & 1991 \\
\hline 52 & E186 & Soil & Thailand NE & 1992 & 121 & E202 & Soil & Thailand NE & 1992 \\
\hline 53 & E193 & Soil & Thailand NE & 1992 & 122 & E221 & Soil & Thailand NE & 1992 \\
\hline 54 & E206 & Soil & Thailand NE & 1992 & 123 & E134 & Soil & Thailand NE & 1991 \\
\hline 55 & $\mathrm{C} 2$ & Soil & Australia & & 124 & E160 & Soil & Thailand NE & 1991 \\
\hline 56 & NT20 & Soil & Burkina Faso & 1973 & 125 & E141 & Soil & Thailand NE & 1991 \\
\hline 57 & 770429 & Soil & Madagascar ${ }^{*}$ & 1977 & 126 & E264 & Soil & Thailand C & 1993 \\
\hline 58 & NT08 & Soil & Niger $^{*}$ & 1973 & 127 & E246 & Soil & Thailand NE & 1992 \\
\hline 59 & 7605 & Manure & France ${ }^{*}$ & 1976 & 128 & E285 & Soil & Thailand C & 1993 \\
\hline 60 & E247 & Soil & Thailand NE & 1992 & 129 & E058 & Soil & Thailand NE & 1990 \\
\hline 61 & E226 & Soil & Thailand NE & 1992 & 130 & E066 & Soil & Thailand NE & 1990 \\
\hline 62 & C7532 & Soil & USA, Oklahoma & & 131 & E286 & Soil & Thailand $\mathrm{C}$ & 1993 \\
\hline 63 & E233 & Soil & Thailand NE & 1992 & 132 & E054 & Soil & Thailand NE & 1990 \\
\hline 64 & E032 & Soil & Thailand NE & 1990 & 133 & E057 & Soil & Thailand NE & 1990 \\
\hline 65 & E203 & Soil & Thailand NE & 1992 & 134 & E061 & Soil & Thailand NE & 1990 \\
\hline 66 & Somredee & Soil & Thailand S & & 135 & E062 & Soil & Thailand NE & 1990 \\
\hline 67 & M7665/91 & Soil & Singapore & 1991 & 136 & E071 & Soil & Thailand NE & 1990 \\
\hline 68 & Kenya & Water & Kenya & 1992 & 137 & E080 & Soil & Thailand NE & 1990 \\
\hline \multirow[t]{2}{*}{69} & D228 & Soil & Australia & $\ldots$ & 138 & E122 & Soil & Thailand NE & 1991 \\
\hline & & & & & 139 & E124 & Soil & Thailand NE & 1991 \\
\hline
\end{tabular}

NE, Northeast; S, South; N, North; C, Central.

..., Date not known.

${ }^{*}$ Received from Institut Pasteur, Paris, France. 
$\begin{array}{llllllllll}1 & 2 & 3 & 4 & 5 & 6 & 7 & 8 & 9 & 10\end{array}$
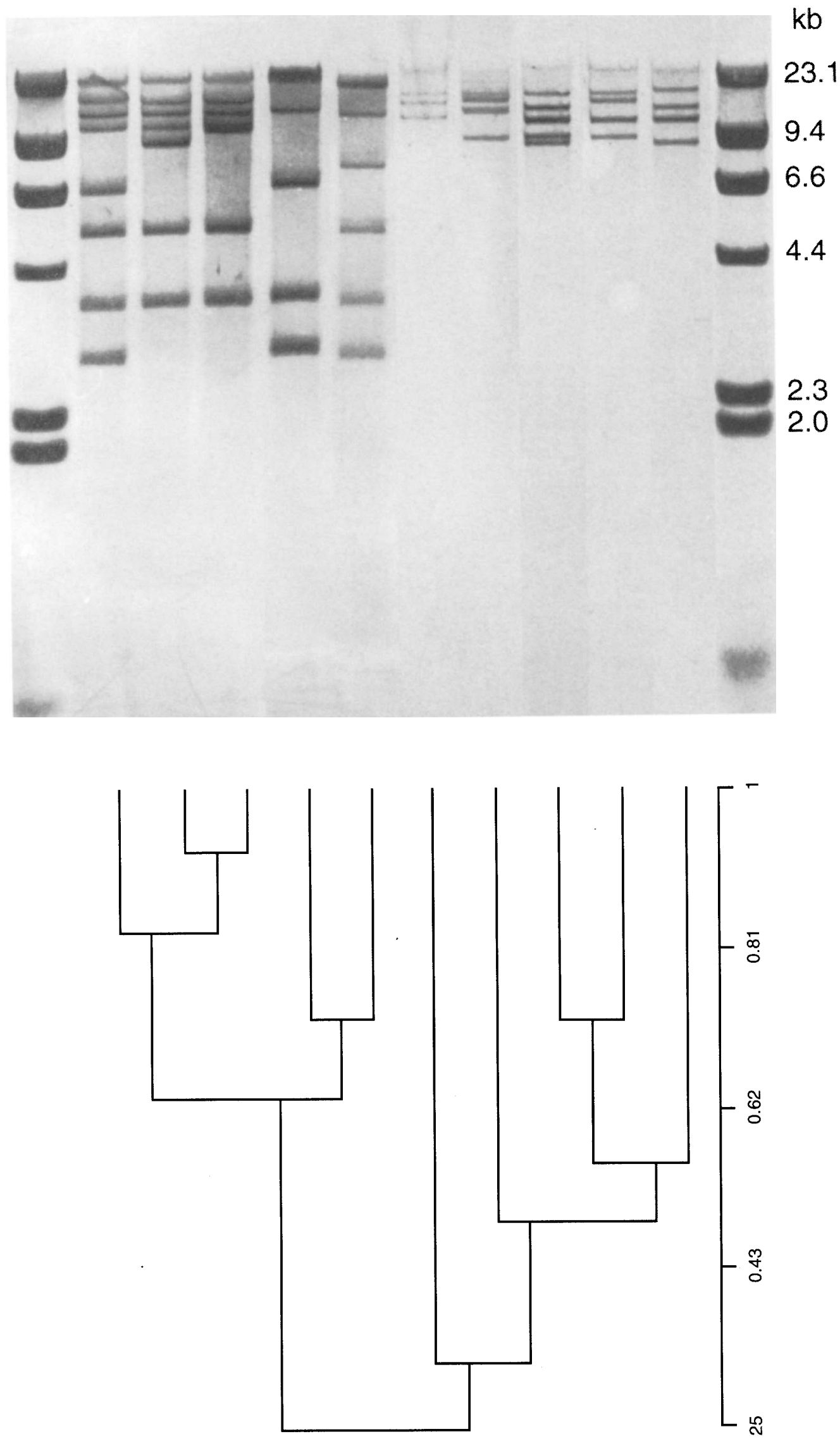

Fig. 1. Hybridisation patterns of group I and II ribotypes. Lanes 1-5, group I; 6-10, group II. The dendrogram shows percentage similarity of patterns within and between ribotype groups. 
Table 2. B. pseudomallei isolates in group I and II ribotypes isolated from environmental and human sources

\begin{tabular}{|c|c|c|}
\hline \multirow[b]{2}{*}{ Ribotype } & \multicolumn{2}{|c|}{ Number $(\%)$ of isolates from } \\
\hline & Environment & Human sources \\
\hline \multicolumn{3}{|l|}{ Group I } \\
\hline 1 & $10 \quad(7.2)$ & $64(28.1)$ \\
\hline 3 & $17(12.8)$ & $45(20.2)$ \\
\hline 4 & $7 \quad(5.6)$ & $14 \quad(6.1)$ \\
\hline 6 & $6 \quad(4.3)$ & $7 \quad(3.1)$ \\
\hline 9 & 1 & $2 \quad(0.9)$ \\
\hline 11 & 0 & $2 \quad(0.9)$ \\
\hline 12 & 0 & $5 \quad(2.2)$ \\
\hline 14 & 1 & $5 \quad(2.2)$ \\
\hline 15 & $6 \quad(4.3)$ & $20 \quad(8.8)$ \\
\hline 16 & $3(2.1)$ & $3 \quad(1.3)$ \\
\hline 19 & 0 & $4 \quad(1.8)$ \\
\hline 26 & $4 \quad(2.8)$ & 3 \\
\hline 27 & 2 (1.4) & $15 \quad(6.6)$ \\
\hline 32 & 0 & $2 \quad(0.9)$ \\
\hline 33 & 0 & $2(0.9)$ \\
\hline 34 & 0 & $7 \quad(3.1)$ \\
\hline 35 & 0 & $2(0.9)$ \\
\hline 37 & $2(1.4)$ & $8 \quad(3.5)$ \\
\hline Unique & $7 \quad(5.0)$ & 0 \\
\hline Unique & 0 & $18 \quad(7.9)$ \\
\hline \multicolumn{3}{|l|}{ Group II } \\
\hline A5 & $27(19.4)$ & 0 \\
\hline A6 & $10(7.1)$ & 0 \\
\hline A8 & $4 \quad(2.8)$ & 0 \\
\hline A9 & $2(1.4)$ & 0 \\
\hline A10 & $3 \quad(2.1)$ & 0 \\
\hline Al1 & $3 \quad(2.1)$ & 0 \\
\hline Unique & $13 \quad(9.3)$ & 0 \\
\hline Untypable & $11 \quad(7.9)$ & 0 \\
\hline Total & 139 & 228 \\
\hline
\end{tabular}

Table 3. Arabinose and ethanol utilisation by B. pseudomallei isolates grouped according to ribotype patterns

\begin{tabular}{|c|c|c|}
\hline \multirow[b]{2}{*}{ Source } & \multicolumn{2}{|c|}{ Number $(\%)$ of isolates showing growth on } \\
\hline & arabinose & ethanol \\
\hline \multicolumn{3}{|l|}{ Environment } \\
\hline All ribotypes & $74 / 139(53.2 \%)$ & $67 / 99(67.7 \%)$ \\
\hline Group I ribotypes & $2 / 66 \quad(3.0 \%)$ & $4 / 26(15.4 \%)^{*}$ \\
\hline Group II ribotypes & $72 / 73 \quad(98.6 \%)^{*}$ & $63 / 73(86.3 \%)$ \\
\hline \multicolumn{3}{|l|}{ Clinical } \\
\hline Group I ribotypes & $0 / 228$ & $1 / 83 \quad(1.2 \%)$ \\
\hline
\end{tabular}

isolates from patients with melioidosis and were of the ara $^{-}$phenotype, whereas group II isolates were invariably $\mathrm{ara}^{+}$.

\section{Discussion}

This study clearly shows the presence of two phylogenetically different populations among isolates that were identified by standard tests as B. pseudomallei. Approximately half of the isolates from soil specimens were of ribotypes similar to those from patients with melioidosis but were markedly different from other environmental isolates. These two ribotype groups also differed in their ability to assimilate arabinose and ethanol.
B. pseudomallei is genomically heterogeneous with respect to rRNA genes and ribotype patterns can be allocated to nearly all strains. Lew and Desmarchelier [9] found 22 patterns among 100 isolates of $B$. pseudomallei from Australia and Southeast Asia and described a similar distribution of hybridisation band sizes to some of the ribotypes described here; however, there is no correspondence between the two numbering systems. In their study, only six environmental isolates from Australia (four soil, two water) were studied and these were grouped in three ribotypes, each of which contained strains from human or animal melioidosis.

It is evident from sequence data that restriction fragment length polymorphisms of rRNA gene provide valuable taxonomic information by which bacteria can be classified. At the species level, these sequences are sufficiently conserved that differences within them often reflect intra-species variation or types. The results obtained here suggest that some environmental organisms classified as $B$. pseudomallei by conventional biochemical and cultural tests are distinguishable from the isolates associated with disease by their ribosomal operons. These genomically atypical isolates are not uniform in ribotype although $c$. $60 \%$ fell into three ribotypes only.

B. pseudomallei is an environmental saprophyte that has been isolated widely from soil and water in Southeast Asia [10,11]. The environment has been suggested as an important source for acquisition of the organism by patients, and this is supported by the finding of similar strain genotypes in isolates from both environmental and clinical sources. However, the relationship between environmental contamination and clinical melioidosis has not been established and it is not known whether an association exists between the distribution of types of $B$. pseudomallei in the environment and clinical presentations of melioidosis.

Species of the genus Burkholderia are distinguished from those of Pseudomonas by their cellular lipid and fatty acid composition, 16S rRNA sequences, DNADNA homology values and phenotypic characteristics [12]. At least 10 different species have been classified within the genes Burkholderia, and these range from strict plant pathogens (e.g., B. solanocearum) to species of medical interest such as $B$. cepacia [13]. $B$. pseudomallei may be confused with $B$. cepacia, as both exhibit relatively similar biochemical test results and have intrinsic resistance to polymyxin and gentamicin. The study by Wuthiekanun et al. [3] clearly showed that ara $^{+}$isolates of $B$. pseudomalle $i$ are not $B$. cepacia and they do not differ sufficiently in biochemical activity from $B$. pseudomallei to warrant their classification in another species by this criterion alone. However, the evidence presented here appears to be inconsistent with a single species concept. Therefore, it will be necessary to examine 
environmental and human isolates of both ribotype groups further for their 16S rRNA sequence similarity and other properties to elucidate their taxonomic status and its relationship to pathogenicity. Furthermore, the significance of arabinose and ethanol utilisation by clinical strains of $B$. pseudomallei is not known, but they may prove to be potential markers of pathogenicity.

We thank the Wellcome Trust (Grant $033262 / \mathrm{Z} / 91 / 2 / 1.5$ ) for their support of the study and Drs. Annette Thomas (Australia), A. L. Tan (Singapore), B. Batchelor (Kenya) and Professor A. Dodin (France) for isolates.

\section{References}

1. Dance DAB. Melioidosis: the tip of the iceberg? Clin Microbiol Rev 1991; 4: 52-60.

2. Chaowagul W, White NI, Dance DAB et al. Melioidosis: a major cause of community-acquired septicemia in Northeastern Thailand. $J$ Infect Dis 1989; 159: 890-899.

3. Wuthiekanun V, Smith MD, Dance DAB, Walsh AL, Pitt TL, White NJ. Biochemical characteristics of clinical and environmental isolates of Burkholderia pseudomallei. J Med Microbiol 1996 45: 408-412.

4. Dance DAB, Wuthiekanun V, Naigowit P, White NJ. Identification of Pseudomonas pseudomallei in clinical practice: use of simple screening tests and API 20NE. J Clin Pathol 1989; 42: 645-649.

5. Clowes RC, Hayes W (eds). Experiments in microbiol genetics. Oxford, Blackwell Scientific Publications. 1968: 184-192.

6. Barrow GI, Feltham RKA (eds). Cowan and Steel's Manual for the identification of medical bacteria, 3rd edn. Cambridge, Cambridge University Press. 1993: 115.

7. Kaufmann ME, Pitcher DG, Pitt TL. Ribotyping of bacterial genomes. In: Chart $\mathrm{H}$ (ed) Methods in practical laboratory bacteriology. Boca Raton, CRC Press. 1994: 123-138.

8. Dice LR. Measures of the amount of ecologic association between species. Ecology 1945; 26: 297-302.

9. Lew AE, Desmarchelier PM. Molecular typing of Pseudomonas pseudomallei: restriction fragment length polymorphisms of rRNA genes. $J$ Clin Microbiol 1993; 31: 533-539.

10. Wuthiekanun V, Smith MD, Dance DAB, White NJ. Isolation of Pseudomonas pseudomallei from soil in north-eastern Thailand. Trans R Soc Trop Med Hyg 1995; 89: 41-43.

11. Van Phung L, Quynh HY, Yabuuchi E, Dance DAB. Pilot study of exposure to Pseudomonas pseudomallei in nothern Vietnam. Trans R Soc Trop Med Hyg 1993; 87: 416.

12. Yabuuchi E, Kosako Y, Oyaizu $\mathrm{H}$ et al. Proposal of Burkholderia gen. nov. and transfer of seven species of the genus Pseudomonas homology group II to the new genus, with the type species Burkholderia cepacia (Palleroni and Holmes 1981) comb. nov. Microbiol Immunol 1992; 36: 1251-1275.

13. Gillis M, Van TV, Bardin R et al. Polyphasic taxonomy in the genus Burkholderia leading to an emended description of the genus and proposition of Burkholderia vietnamiensis sp. nov. for $\mathrm{N}_{2}$-fixing isolates from rice in Vietnam. Int $J$ Syst Bacteriol 1995; 45: 274-289. 